\title{
Correction to: Walking Stabilization Control for Humanoid Robots on Unknown Slope Based on Walking Sequences Adjustment
}

\author{
Jiatao Ding ${ }^{1} \cdot$ Yang Wang $^{1} \cdot$ Minghui Yang $^{1} \cdot$ Xiaohui Xiao $^{1}$ \\ Published online: 7 February 2018 \\ (C) Springer Science+Business Media B.V., part of Springer Nature 2018 \\ Correction to: J Intell Robot Syst \\ https://doi.org/10.1007/s10846-017-0685-4
}

The original version of this manuscript unfortunately contained errors.

On page 14, "Grant No. 51175383" should be "Grant No. 51675385." The correct line should read: This research is supported by the National Foundation of China (Grant No. 51675385).

On page 16, the line "Jiatao Ding received the B.S. degree in Mechanical Design, Manufacture and Automation in 2010 from Wuhan University, China" should be "Jiatao Ding received the B.S. degree in Mechanical Design, Manufacture and Automation in 2014 from Wuhan University, China."

The online version of the original article can be found at https://doi.org/10.1007/s10846-017-0685-4.

Xiaohui Xiao

xhxiao@whu.edu.cn

Jiatao Ding

jtdingx@163.com

Yang Wang

wangyang.hf1987@whu.edu.cn

Minghui Yang

mhyang@whu.edu.cn

1 Department of Mechanical Engineering, School of Power and Mechanical Engineering, Wuhan University, No8, South Donghu Road, Wuchang district, Wuhan, 430072, China 